\title{
Evidence against an effect of grouping by spectral regularity on the perception of virtual pitch
}

\author{
Valter Ciocca ${ }^{\text {a) }}$ \\ Department of Speech and Hearing Sciences, University of Hong Kong, Hong Kong
}

(Received 13 January 1999; revised 21 June 1999; accepted 9 July 1999)

\begin{abstract}
Two experiments investigated the role of the regularity of the frequency spacing of harmonics, as a separate factor from harmonicity, on the perception of the virtual pitch of a harmonic series. The first experiment compared the shifts produced by mistuning the 3rd, 4th, and 5th harmonics in the pitch of two harmonic series: the odd- $\mathrm{H}$ and the all-H tones. The odd- $\mathrm{H}$ tone contained odd harmonics 1 to 11, plus the 4th harmonic; the all-H tone contained harmonics 1 to 12 . Both tones had a fundamental frequency of $155 \mathrm{~Hz}$. Pitch shifts produced by mistuning the 3rd harmonic, but not the 4th and 5th harmonics, were found to be significantly larger for the odd-H tone than for the all-H tone. This finding was consistent with the idea that grouping by spectral regularity affects pitch perception since an odd harmonic made a larger contribution than an adjacent even harmonic to the pitch of the odd-H tone. However, an alternative explanation was that the 3rd mistuned harmonic produced larger pitch shifts within the odd- $\mathrm{H}$ tone than the 4th mistuned harmonic because of differences in the partial masking of these harmonics by adjacent harmonics. The second experiment tested these explanations by measuring pitch shifts for a modified all-H tone in which each mistuned odd harmonic was tested in the presence of the 4th harmonic, but in the absence of its other even-numbered neighbor. The results showed that, for all mistuned harmonics, pitch shifts for the modified all-H tone were not significantly different from those for the odd- $\mathrm{H}$ tone. These findings suggest that the harmonic relations among frequency components, rather than the regularity of their frequency spacing, is the primary factor for the perception of the virtual pitch of complex sounds. (C) 1999 Acoustical Society of America. [S0001-4966(99)00611-6]
\end{abstract}

PACS numbers: 43.66.Ba, 43.66.Hg [SPB]

\section{INTRODUCTION}

In a series of studies, Roberts and his colleagues have demonstrated that the auditory grouping of a frequency component into a complex tone is affected by how well the component fits in with the spectral pattern of the complex tone (Roberts and Bregman, 1991; Roberts and Bailey, 1993a, b). Roberts and Bregman (1991) asked subjects to rate how clearly individual even- or odd-numbered harmonics could be "heard out" of a complex tone composed only of odd harmonics. Subjects gave higher perceived clarity ratings to even than odd harmonics, in spite of the fact that even- and odd-numbered harmonics had a common fundamental frequency $(F 0)$. The greater perceptual salience of even harmonics was surprising given that the partial masking of an even harmonic was greater than that of its odd-numbered neighbors in complex stimuli composed of odd harmonics. The authors suggested that even-numbered harmonics were more easily segregated than odd harmonics because the former violated the pattern of spectral regularity of the oddharmonic series. Roberts and Bregman also found that the effect of spectral regularity was $F 0$ dependent, since it was observed for F0's of 100 and $200 \mathrm{~Hz}$, but not $400 \mathrm{~Hz}$. Roberts (1998) replicated earlier perceived clarity ratings results by using a two-internal forced choice (2IFC) procedure and by obtaining a difference in $d^{\prime}$ scores between even- and odd-numbered harmonics.

${ }^{a)}$ Electronic mail: vciocca@hkusua.hku.hk
Roberts and Bailey (1996) demonstrated that the effects of spectral regularity were not restricted to frequency components that are harmonically related, but also occurred for inharmonic complex tones whose components were arranged in a regular spectral pattern. Roberts and Brunstrom (1998) provided further evidence for the role of spectral regularity as a grouping factor by investigating the perceptual integration of mistuned frequency components within complex tones (see Hartmann et al., 1990). Roberts and Brunstrom found that the perceptual segregation and the perceived pitch shifts of individual mistuned components were similar for harmonic and inharmonic, but spectrally regular, complex tones. These results, which were interpreted as evidence for the role of spectral regularity as a grouping principle distinct from harmonicity (Roberts and Bailey, 1996; Roberts and Brunstrom, 1998), raise the interesting possibility that spectral regularity might also affect the integration of frequency components into a single "virtual" (or "residue") pitch (the term "pitch" will be used as synonymous of "virtual pitch," hereafter).

Several models of virtual pitch perception are based on the assumption that the pitch of complex tones is determined by the frequencies of the resolved, low-numbered harmonics (see, e.g., Goldstein, 1973; Terhardt, 1974). In order to obtain an accurate estimate of the pitch of a complex sound in the presence of other simultaneous sounds, the auditory system must decide which frequency components should contribute to the pitch of the complex sound. This decision is likely to be made on the basis of several auditory scene 
analysis (or auditory grouping) principles (see, for example, Bregman, 1990; Darwin and Ciocca, 1992). Several studies demonstrated that the integration of frequency components into a single pitch is affected by grouping factors such as onset time and ear of presentation (Darwin and Ciocca, 1992; Ciocca and Darwin, 1993), frequency modulation (Darwin et al., 1994), and sequential grouping by frequency proximity (Darwin et al., 1995). For example, Darwin and Ciocca (1992) found that a mistuned component that starts $300 \mathrm{~ms}$ before the onset of the remainder of a harmonic series does not make a contribution to the pitch of the series. They also found that when the mistuned component and the harmonic series were presented to opposite ears the contribution of the mistuned component to the pitch of the harmonic series was reduced, albeit only slightly. One of the grouping principles that has been shown to affect pitch perception is the principle of harmonicity: frequency components that are harmonics of the same fundamental frequency would contribute to a single virtual pitch. The operation of this principle has been likened to a "harmonic sieve" whose "slots" are centered on the harmonic frequencies of a fundamental frequency (Duifhuis et al., 1982). Moore et al. (1985) provided evidence for the existence of such a mechanism by showing that frequency components contribute to the pitch of a harmonic series as long as their frequency is not too far from one of the harmonic frequencies. Moore et al. showed that mistuned harmonics made the largest contribution to the pitch of a harmonic series for mistunings equal or smaller than $\pm 3 \%$ of the harmonic frequencies. For mistunings larger than $\pm 3 \%$, the contribution of the mistuned harmonics was gradually reduced and became very small for mistunings of $\pm 8 \%$ (the largest mistuning used).

If spectral regularity is a grouping factor which is distinct from harmonicity, then it is possible that pitch perception processes might exploit the pattern of spectral regularity of frequency components to assign them to a single virtual pitch. This investigation attempted to find evidence for this hypothesis by using the "pitch matching" paradigm developed by Moore et al. (1985). In this paradigm, listeners are asked to match the pitch of two harmonic series, the target and the matching complexes. Across trials, a low-numbered harmonic of the target complex can be mistuned by various amounts; the amount of mistuning is fixed within each trial. Pitch matches are carried out by adjusting the $F 0$ of the matching complex as many times as necessary for listeners to achieve a satisfactory match. Typical results show that when the frequency of the mistuned harmonic is higher than the harmonic frequency ("positive" mistuning), the matched $F 0$ of the target is also higher than the matched $F 0$ of the same target complex whose harmonics are not mistuned. Pitch shifts of similar size but opposite direction occur for "negative" mistunings. Since it has been shown that changes in the matched $F 0$ of the matching complex reflect changes in the virtual pitch of the target complex (Moore, 1987), this procedure is ideally suited for studying the conditions under which a frequency component is integrated into the pitch of a complex tone. The present study investigated the effects of spectral regularity on pitch perception by measuring the pitch shifts for the target complex as a function of the harmonic number of the mistuned harmonic, and of the spectral content of the target complex.

\section{EXPERIMENT 1}

If grouping by spectral regularity affects the perception of virtual pitch, then frequency components that do not fit in with the pattern of frequency spacing of a complex tone should make a relatively small contribution to the pitch of that tone. Therefore, odd-numbered mistuned harmonics should produce larger pitch shifts than adjacent evennumbered harmonics in the pitch of a harmonic series composed of primarily odd harmonics. Moreover, mistuned odd harmonics, but not even-numbered ones, should produce larger pitch shifts in an odd-harmonic series than in a series that contains both odd and even harmonics.

\section{A. Method}

On each trial, the subjects adjusted the $F 0$ of a harmonic series (matching complex) so that the pitch of that series matched the pitch of a target complex. Two target complexes were used in the experiment, the "odd- $\mathrm{H}$ " tone and the "all$\mathrm{H}$ " tone. The all-H tone contained 12 consecutive harmonics (1st to 12 th) of a $155-\mathrm{Hz} F 0$; this target complex was the same as the one used in previous studies by Darwin and his colleagues (see, e.g., Darwin and Ciocca, 1992). The odd-H tone had the same fundamental frequency as the all-H tone, but was composed of the odd harmonics 1 to 11, plus the 4th harmonic. A $155-\mathrm{Hz} F 0$ was selected because the effect of spectral regularity was observed for fundamental frequencies of 100 and $200 \mathrm{~Hz}$ (Roberts and Bregman, 1991). Moore and his colleagues (1985) showed that the contribution of a mistuned harmonic to the pitch of a complex tone that contained both odd and even harmonics depended on the harmonic number of the mistuned component. They found that the largest pitch shifts were obtained when the 3rd and 4th harmonics were mistuned and that, for fundamental frequencies above $100 \mathrm{~Hz}$, pitch shifts produced by mistuning the 5th and higher harmonics were very small for two out of three subjects. Following these findings, the present study tested the role of spectral regularity in pitch perception by comparing the relative size of the pitch shifts produced by mistuning the 3rd, 4th, and 5th harmonics in the two target complexes. These harmonics could be mistuned by $\pm 3 \%$ of the harmonic frequency. This amount of mistuning was selected because it was found to produce the largest pitch shifts in a 155-Hz F0 harmonic series (Darwin and Ciocca, 1992). The mistuned 3rd harmonic had a frequency of 451 or $479 \mathrm{~Hz}$ ( - or $+3 \%$ mistuning, respectively); the mistuned 4th harmonic had a frequency of 600 or $640 \mathrm{~Hz}$, and the mistuned 5th harmonic had a frequency of 752 or $798 \mathrm{~Hz}$. Within a trial, only one of the three harmonics was mistuned. The 4th harmonic was included in the odd- $\mathrm{H}$ tone when the 3rd and 5th harmonics were mistuned in order to ensure that pitch shifts for odd and even harmonics were measured with targets that contained the same frequency components, except for the mistuned harmonic. Two matching complexes were used in order to minimize timbre differences that might have affected the adjustment of the pitch of the matching complex to that of the target complex (see, for example, Moore and 
Glasberg, 1990; Singh and Hirsh, 1992). When the target was the all-H tone, the matching complex consisted of harmonics 1 to 12; the matching complex employed with odd- $\mathrm{H}$ tone was composed of the 1st, 3rd, 4th, 5th, 7th, 9th, and 11th harmonics. None of the harmonics of the matching complexes was mistuned. Subjects could adjust the $F 0$ of the matching complexes between 151 and $159 \mathrm{~Hz}$ (155 $\pm 4 \mathrm{~Hz})$.

Within each trial, the matching complex followed the target complex after a 500-ms silent interval. At the beginning of each trial, the fundamental frequency of the matching complex was set to a random value selected from the permitted range $(155 \pm 4 \mathrm{~Hz})$, and the cursor was displayed at the center of a computer screen. Subjects adjusted the frequency of the matching complex by moving a roller-ball up or down, which moved upward or downward the position of the cursor on the screen. The fundamental frequency of the matching complex varied in steps of $0.0066 \mathrm{~Hz}$ per pixel, which corresponded to a variation of $3.2 \mathrm{~Hz}$ for a complete excursion of the cursor from the top to the bottom of the screen (see Darwin and Ciocca, 1992). Subjects could make as many adjustments as needed to achieve a satisfactory match by clicking the mouse button after each adjustment. When the match was satisfactory, subjects pressed the "return" key and proceeded to the next trial. Since the mistuned harmonics could be heard out as individual pure tones, listeners were instructed to ignore any pure tones they might hear, and to focus their attention on the pitches of the two complex tones.

To summarize, the experiment consisted of 12 stimuli: two mistunings $(-3 \%,+3 \%$ of the harmonic frequency) by three harmonic numbers (3rd, 4th, and 5th harmonic) by two types of target complex (all-H and odd-H). Five matches were obtained for each stimulus, thus giving a total of 60 trials (12 stimuli by 5 matches). The order of presentation was randomized within each block of 12 stimuli. The experiment was completed within either one or two sessions. Each session took from 1 to $2 \mathrm{~h}$ to complete, including rest breaks.

All components were presented to the left ear at the same amplitude (corresponding to $58 \mathrm{~dB}$ SPL, for a $1000-\mathrm{Hz}$ tone). All components started at sine phase. The mistuned component, the target complex, and the matching complex had a total duration of $90 \mathrm{~ms}$, including 5-ms rise/fall raisedcosine ramps. This duration was the same as that used in previous pitch matching experiments (Darwin and Ciocca, 1992; Ciocca and Darwin, 1993). Sounds were presented through Sennheiser HD250 Linear headphones in a singlewalled sound-attenuating booth (Industrial Acoustics) that was located in a sound-treated room. Sounds were synthesized in real-time at $44.1 \mathrm{kHz}$ using custom software (Russell and Darwin, 1991) written for the 56001 processor of the Digidesign Audiomedia II board, and output through that board's 16-bit DACs and anti-aliasing filters. The board was attached to a PowerMacintosh 7100/66 computer which controlled the experiment.

Seven subjects (four undergraduate students and three university staff, including the author) participated in the experiment. Two of the subjects had received musical training. All had participated in previous pitch matching experiments

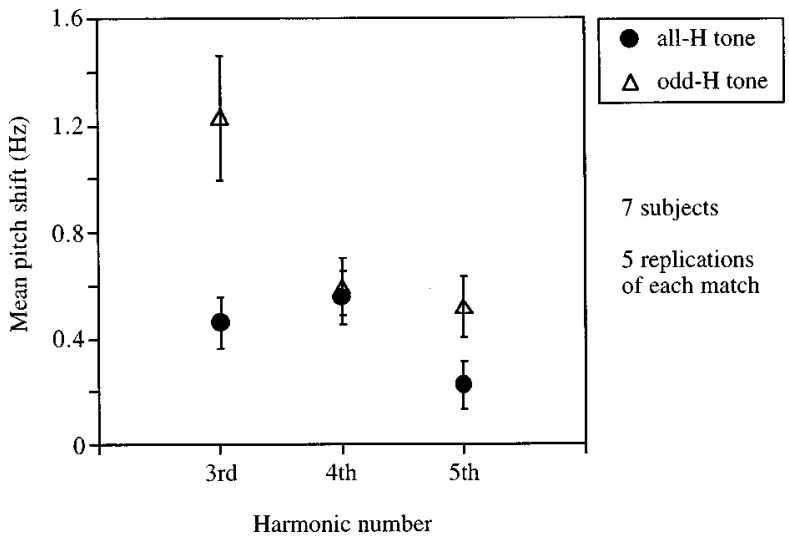

FIG. 1. Mean pitch shifts with standard error bars for the odd-H tone (open triangles) and the all-H tone (filled circles), as a function of the harmonic number of the mistuned component, in experiment 1 .

and had pure tone thresholds within $15 \mathrm{~dB}$ HL around the stimulus frequencies used in the experiment.

\section{B. Results and discussion}

The effects of the mistuning of the three harmonics (3rd, 4th, and 5th) on the pitch of the target complex were measured by calculating the average of the difference between the pitch matches for positive and negative mistunings (mean pitch shifts) for each mistuned harmonic and each subject. The mean pitch shifts for each mistuned harmonic and target complex, averaged across the seven subjects, are displayed in Fig. 1.

A two-way ANOVA with repeated measures was applied to the mean pitch shifts. The factors were the "harmonic number" of the mistuned harmonic (3rd, 4th, or 5th) and the "type of target" (all-H or odd-H tone). The main effect of harmonic number was statistically significant, $F(2,12)=8.33, p<0.01$. Significantly larger mean pitch shifts were observed for the odd-H tone than for the all-H tone (main effect of "target type," $F(1,6)=6.76, p$ $<0.05)$. The "target type" by "harmonic number" interaction shows that the pattern of pitch shifts for the two target complexes was significantly different, $F(2,12)=5.19, p$ $<0.05$. The nature of this interaction can be illustrated by comparing the pitch shifts obtained with odd- $\mathrm{H}$ and all- $\mathrm{H}$ targets for each harmonic number. Pitch shifts were significantly larger for the odd- $\mathrm{H}$ than for the all- $\mathrm{H}$ tone for the 3rd harmonic (planned comparison, $p<0.001$ ). These results support the hypothesis that odd harmonics are more strongly integrated into the pitch of odd-H tones than even harmonics, and that grouping by spectral regularity affects virtual pitch perception. According to the same hypothesis, one would also have expected that pitch shifts produced by the 4th harmonic were significantly smaller for the odd- $\mathrm{H}$ than for the all-H tone. However, this comparison was not statistically significant (planned comparison, $p>0.05$ ). Although pitch shifts for the 5th harmonic were more than twice as large for the odd- $\mathrm{H}$ tone $(0.52 \mathrm{~Hz})$ than for the all- $\mathrm{H}$ tone $(0.23 \mathrm{~Hz})$, this difference failed to reach statistical significance (planned comparison, $p=0.093$ ). The failure to find a significant difference in the size of pitch shifts for the 5th harmonic in 
odd-H and all-H tones was probably due to the fact that pitch shifts for this component were small with respect to the variance in the data (see also Moore et al., 1985).

To conclude, the finding that pitch shifts produced by a mistuned 3rd harmonic were larger for the odd- $\mathrm{H}$ tone than for the all-H tone supports the idea that grouping by spectral regularity affects virtual pitch perception. The failure to observe a decrease in pitch shifts for a mistuned 4th harmonic within the odd-H tone, relative to the shifts produced by the same mistuned harmonic in the all-H, could be interpreted as evidence against a role of spectral regularity in pitch perception. However, the latter result could also be taken as evidence in favor of an effect of spectral regularity if one considers that the contribution of a mistuned component to the pitch of a harmonic series might be a function of the total number of partials in the harmonic series. ${ }^{1}$ Moore and his colleagues demonstrated that harmonics 1 to 6 are dominant for the perception of the virtual pitch of harmonic series whose $F 0$ 's are in the $100-200-\mathrm{Hz}$ frequency region (Moore et al., 1985; Moore and Glasberg, 1990). In this experiment, the odd-H tone contained four harmonics in the dominance region while the all-H tone contained six harmonics. For this reason, one might have expected that the pitch shifts produced by each mistuned component should have been larger for the odd-H than for the all-H tone. The failure to observe an increase in the pitch shifts produced by the 4th harmonic in the odd-H tone, relative to the all-H tone, could be interpreted as an effect of the perceptual segregation of the 4th harmonic from the remaining odd harmonics in the odd- $\mathrm{H}$ tone.

An alternative explanation for the difference in the pitch shifts produced by the 3rd and 4th harmonics within the odd-H tone is based on the fact that the 3rd harmonic was flanked by only one adjacent harmonic (i.e., the 4th harmonic). By contrast, the 4th harmonic was flanked by two adjacent harmonics (the 3rd and the 5th harmonics) both in the odd-H and the all-H tones. ${ }^{2}$ Moore et al. (1985) showed that the contribution of the 2 nd harmonic to the virtual pitch of a $200-\mathrm{Hz} F 0$, all-H tone decreased as the level of the harmonic was reduced relative to the level of adjacent harmonics. Therefore, it is possible that the greater amount of masking of the 4th harmonic could explain its reduced contribution to the pitch of the odd- $\mathrm{H}$ tone, relative to the pitch shifts obtained with the 3rd harmonic. Moreover, because of the upward spread of masking, the partial masking of the 5th harmonic by the 4th harmonic should be greater than the partial masking of the 3rd harmonic by the 4th harmonic. This partial masking difference could also have contributed to the finding that pitch shifts for the 3rd mistuned harmonic were significantly larger for the odd- $\mathrm{H}$ than for the all-H tone while no difference was found for the 5 th mistuned harmonic between the pitch shifts for the odd- $\mathrm{H}$ and the all-H tones.

\section{EXPERIMENT 2}

This experiment was conducted to test whether the results of experiment 1 were likely to be due to differences in masking rather than spectral regularity. To do this, the pitch shifts produced in an odd-H tone were compared with those obtained in a target complex which contained all the harmon- ics up to the 11th, except for the 2 nd or the 6th harmonic (modified all-H tone). The 2 nd harmonic was removed from the all-H tone when the 3rd harmonic was mistuned; the 6th harmonic was missing when the 5th harmonic was mistuned. These stimuli were used to compare pitch shifts in the two target complexes under the same amount of masking by adjacent harmonics. If spectral regularity does not affect pitch perception, then the pitch shifts in the two target complexes should be identical for each mistuned harmonic. By contrast, if the integration of harmonics into a single pitch is affected by spectral regularity, the pattern of pitch shifts observed in experiment 1 as a function of the harmonic number of the mistuned harmonic should be obtained with the odd- $\mathrm{H}$, but not with the modified all-H tone.

\section{A. Method}

The harmonic numbers and the amounts of mistuning of the mistuned harmonics were identical to those of experiment 1 , except that an in-tune condition ( $0 \%$ mistuning) was included. Two target complexes were used in the experiment: the odd-H tone and the "modified all-H tone." The former was identical to the odd-H tone used in the first experiment. The latter contained harmonics 1 to 11 of a $155-\mathrm{Hz}$ $F 0$, except that one harmonic could be missing. The missing harmonic was harmonic 2 (when the 3rd harmonic was mistuned), or harmonic 6 (when the 5th harmonic was mistuned). No harmonic was missing when the 4th harmonic of the modified all-H tone was mistuned. When the modified all-H tone was presented in a trial, the matching complex consisted of the same harmonics as the modified all-H tone within that trial. For example, if the 3rd harmonic was mistuned within the modified all-H tone, the matching complex contained harmonic 1 and harmonics 3-11 (harmonic 2 was missing). The matching complex employed with the odd- $\mathrm{H}$ tone was composed of the 1st, 3rd, 4th, 5th, 7th, 9th, and 11th harmonics. The pitch matching procedure was identical to that employed in experiment 1 .

To summarize, the experiment consisted of 18 stimuli: three mistunings $(-3 \%, 0 \%,+3 \%$ of the harmonic frequency) by three harmonic numbers (3rd, 4th, and 5 th harmonic) by two types of target complexes (modified all-H and odd-H tones). Subjects completed between five and eight matches for each stimulus; for each subject, all stimuli were matched an equal number of times. The order of presentation was randomized within each block of 18 stimuli. The experiment was completed within either one or two sessions. Each session took from 1 to $2 \mathrm{~h}$ to complete, including rest breaks.

The stimuli were presented at the same level and had the same duration as in the first experiment. The software/ hardware experimental setup was the same as in the previous experiment.

Six listeners, including the author, took part in the experiment; three of the listeners were musically trained. All subjects had pure tone thresholds within $15 \mathrm{~dB}$ HL around the stimulus frequencies used in the experiment. Five listeners had participated in experiment 1. 


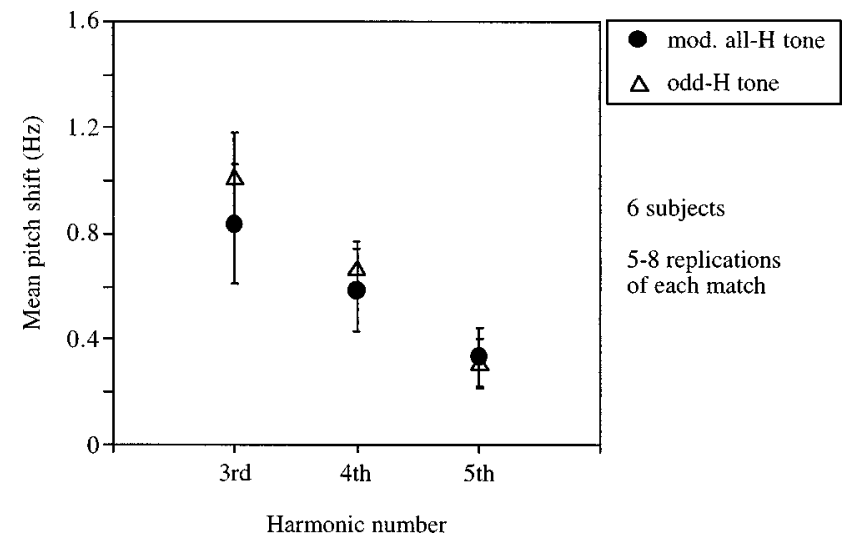

FIG. 2. Mean pitch shifts with standard error bars for the odd-H tone (open triangles) and the modified all-H tone (filled circles), as a function of the harmonic number of the mistuned component, in experiment 2.

\section{B. Results}

The mean pitch shifts for each mistuned harmonic and target complex, averaged across the six subjects, are displayed in Fig. 2. This figure shows that the pattern of pitch shifts for the odd- $\mathrm{H}$ and the all-H targets was very similar. A two-way ANOVA with repeated measures was applied to the mean pitch shifts. The factors were the "harmonic number", (3rd, 4th, or 5th) of the mistuned harmonic and the "type of target" (modified all-H or odd-H tone). The main effect of harmonic number was statistically significant, $F(2,10)$ $=6.04, p<0.05$. This effect replicated the results of experiment 1 . All other effects were not statistically significant. For all harmonics, the planned comparisons between the pitch shifts for the modified all-H and the odd-H target complexes failed to reach statistical significance. The fact that pitch shifts produced by the 3rd harmonic in the odd- $\mathrm{H}$ tone were slightly smaller in this experiment $(1.02 \mathrm{~Hz}$; see Fig. 2) than in the previous one $(1.23 \mathrm{~Hz}$; see Fig. 1) might have contributed in reducing the difference between pitch shifts produced by the 3rd harmonic in the present experiment. However, in both experiments pitch shifts in the odd-H tone were significantly larger for harmonic 3 than for harmonic 4 (NewmanKeuls post hoc test, $p<0.05)$. For this reason, it seems unlikely that a decrease in pitch shifts for the 3rd harmonic in the odd-H tone in the present experiment was the major reason for the failure to find a statistically significant difference between pitch shifts for the odd- $\mathrm{H}$ and the modified all-H tones.

As discussed previously (Sec. IB), the contribution of a mistuned harmonic to the pitch of the target complex might have been affected by the number of partials (up to the sixth) which were present in the target complexes. In this experiment, the odd- $\mathrm{H}$ tone still contained four partials, while the modified all-H tone contained either five or six of the harmonics (1-6) which were found to be dominant for pitch perception (Moore et al., 1985; Moore and Glasberg, 1990). In spite of this difference, neither the main effect of the type of target nor the pairwise comparisons between pitch shifts in the two target complexes for each mistuned component were statistically significant. Therefore, it seems likely that the results of this experiment were not affected by differ- ences in the number of harmonics which were present in the target complexes.

In conclusion, the results of this experiment support the hypothesis that the differences in the pattern of pitch shifts for odd-H and all-H tones observed in experiment 1 can be attributed to the effects of partial masking, not to the effects of grouping by spectral regularity.

\section{GENERAL DISCUSSION}

In the first experiment, pitch shifts produced by the 3rd harmonic in the odd- $\mathrm{H}$ tone were significantly larger than shifts produced by the same mistuned harmonic in the all- $\mathrm{H}$ tone. This difference was not statistically significant when the 4th harmonic was mistuned. These results could have been interpreted as evidence for the effect of spectral regularity on pitch perception. The second experiment showed that when the same adjacent harmonics flanked the mistuned harmonics, pitch shifts for the odd- $\mathrm{H}$ tone and for the modified all-H tone were similar for all mistuned harmonics. These findings demonstrated that the difference in the pitch shifts for odd and even harmonics found in experiment 1 was probably due to differences in partial masking, and not to effects of spectral regularity.

Roberts and Bailey (1996) suggested that grouping by harmonicity could be considered as a special case of grouping by spectral regularity, and that some of the grouping effects normally attributed to harmonicity could, in fact, be cases of grouping by spectral regularity. The results of the current study suggest that grouping by spectral regularity does not affect the integration of frequency components into a single virtual pitch. In the case of pitch perception, it is likely that the assignment of simultaneous frequency components to a single pitch is determined mainly on the basis of the harmonic relationships among components ("harmonic sieve" mechanism; Duifhuis et al., 1982; Moore et al., 1985). Harmonics of the same fundamental frequency would pass through the sieve, and make a contribution to the pitch of a harmonic series, independently of whether they fit with the regularity of the spectral pattern of the series.

This conclusion is consistent with the idea that the perceptual segregation of individual frequency components and pitch perception processes operate on the basis of different grouping constraints. In fact, Moore and his colleagues found that frequency components can contribute to the pitch of a harmonic series for mistunings of up to $8 \%$ of the harmonic frequency (Moore et al., 1985), even though they are perceptually segregated from the series when they are mistuned by about $1 \%$ or more (Moore et al., 1986). Therefore, it appears that spectral regularity might affect the perceptual separation of a frequency component but not its integration into the pitch of a harmonic series. Although the current data argue against the effects of grouping by spectral regularity on pitch perception, it is possible that this grouping principle plays a role not only in the perceptual segregation of frequency components, but also in the processing of other characteristics of perceived sounds, such as timbre and perceived location. The effects of grouping by spectral regularity on these perceptual attributes of sounds have yet to be investigated. 


\section{ACKNOWLEDGMENTS}

The author would like to thank Brian Roberts, Robert Carlyon, and Sid Bacon for providing helpful comments on the manuscript and Duncan Lam, Chloe Lam, and Ida Tsui for helping with data collection. The study was funded through grant H.K.R.G.C. HKU 362/94M awarded to the author.

${ }^{1}$ The author would like to thank Brian Roberts for suggesting this interpretation of the results of experiment 1 .

${ }^{2}$ Within the odd-H tone the equivalent regular band width (ERB) separation between the 3rd harmonic and the 1st harmonic (the adjacent, lowerfrequency harmonic), calculated using Glasberg and Moore's (1990) formulas, was 5.5 ERB units. Within the all-H tone, the 3rd harmonic and the adjacent, lower-frequency harmonic (harmonic 2) were separated by 2.39 ERB units. Therefore, within the all-H tone, the partial masking of the 3rd harmonic by adjacent harmonics was probably larger than within the odd- $\mathrm{H}$ tone. Moreover, the ERB separation between harmonics 3 and 4 was 1.87 ERB units. Therefore, the partial masking of the 4th harmonic was larger than that of the 3rd harmonic within an odd-H tone.

Bregman, A. S. (1990). Auditory Scene Analysis: The Perceptual Organisation of Sound (MIT, Cambridge, MA).

Ciocca, V., and Darwin, C. J. (1993). "Effects of onset asynchrony on pitch perception: Adaptation or grouping?"' J. Acoust. Soc. Am. 93, 28702878.

Darwin, C. J., and Ciocca, V. (1992). "Grouping in pitch perception: Effects of onset asynchrony and ear of presentation of a mistuned component,"' J. Acoust. Soc. Am. 91, 3381-3390.

Darwin, C. J., Ciocca, V., and Sandell, G. J. (1994). "Effects of frequency and amplitude modulation on the pitch of a complex tone with a mistuned harmonic,"' J. Acoust. Soc. Am. 95, 2631-2636.

Darwin, C. J., Hukin, R. W., and Al-Khatib, B. Y. (1995). "Grouping in pitch perception: Evidence for sequential constraints,' J. Acoust. Soc. Am. 98, 880-885.

Duifhuis, H., Willems, L. F., and Sluyter, R. J. (1982). "Measurement of pitch in speech: an implementation of Goldstein's theory of pitch perception," J. Acoust. Soc. Am. 71, 1568-1580.

Glasberg, B. R., and Moore, B. C. J. (1990). "Derivation of auditory filter shapes from notched-noise data," Hear. Res. 47, 103-138.

Goldstein, J. L. (1973). “An optimum processor theory for the central for- mation of the pitch of complex tones," J. Acoust. Soc. Am. 54, 14961516.

Hartmann, W. M., McAdams, S., and Smith, B. K. (1990). "Hearing a mistuned harmonic in an otherwise periodic complex tone," J. Acoust. Soc. Am. 88, 1712-1724.

Moore, B. C. J. (1987). "The perception of inharmonic complex tones," Auditory Processing of Complex Sounds, edited by W. A. Yost and C. S. Watson (Erlbaum, Hillsdale, NJ), pp. 180-189.

Moore, B. C. J., and Glasberg, B. R. (1990). "Frequency discrimination of complex tones with overlapping and non-overlapping harmonics," J. Acoust. Soc. Am. 87, 2163-2177.

Moore, B. C. J., Glasberg, B. R., and Peters, R. W. (1985). "Relative dominance of individual partials in determining the pitch of complex tones," J. Acoust. Soc. Am. 77, 1853-1860.

Moore, B. C. J., Glasberg, B. R., and Peters, R. W. (1986). "Thresholds for hearing mistuned partials as separate tones in harmonic complexes," $\mathrm{J}$. Acoust. Soc. Am. 80, 479-483.

Roberts, B. (1998). "Effects of spectral pattern on the perceptual salience of partials in harmonic and frequency-shifted complex tones: A performance measure,"' J. Acoust. Soc. Am. 103, 3588-3596.

Roberts, B., and Bailey, P. J. (1993a). "Spectral pattern and the perceptual fusion of harmonics: I. The role of temporal factors," J. Acoust. Soc. Am. 94, 3153-3164.

Roberts, B., and Bailey, P. J. (1993b). "Spectral pattern and the perceptual fusion of harmonics: II. A special status for added components?" J. Acoust. Soc. Am. 94, 3165-3177.

Roberts, B., and Bailey, P. J. (1996). "Spectral regularity as a factor distinct from harmonic relations in auditory grouping," J. Exp. Psychol. 22(3), 604-614.

Roberts, B., and Bregman, A. S. (1991). "Effects of the pattern of spectral spacing on the perceptual fusion of harmonics," J. Acoust. Soc. Am. 90, 3050-3060.

Roberts, B., and Brunstrom, J. M. (1998). "'Perceptual segregation and pitch shifts of mistuned components in harmonic complexes and in regular inharmonic complexes,"' J. Acoust. Soc. Am. 104, 2326-2338.

Russell, P., and Darwin, C. J. (1991). "Real-time synthesis of complex sounds on a Mac II with 56001 DSP chip," Br. J. Audiol. 25, 59-60.

Singh, P. G., and Hirsh, I. J. (1992). "Influence of spectral locus and $F 0$ changes on the pitch and timbre of complex tones," J. Acoust. Soc. Am. 92, 2650-2661.

Terhardt, E. (1974). "Pitch, consonance, and harmony," J. Acoust. Soc. Am. 55, 1061-1069. 